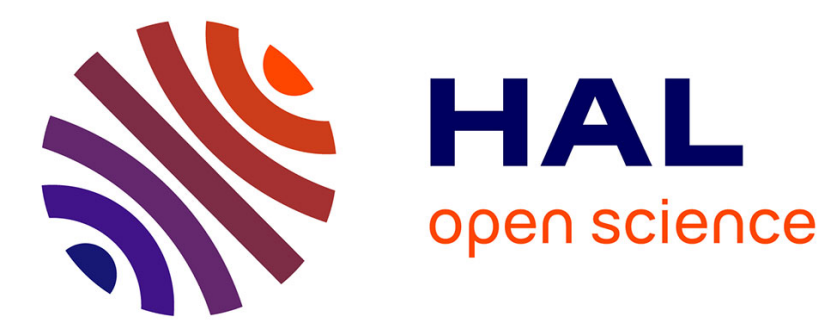

\title{
Van Hove singularities and continued fraction coefficients
}

\author{
C.H. Hodges
}

\section{To cite this version:}

C.H. Hodges. Van Hove singularities and continued fraction coefficients. Journal de Physique Lettres, 1977, 38 (9), pp.187-189. 10.1051/jphyslet:01977003809018700 . jpa-00231356

\section{HAL Id: jpa-00231356 https://hal.science/jpa-00231356}

Submitted on 1 Jan 1977

HAL is a multi-disciplinary open access archive for the deposit and dissemination of scientific research documents, whether they are published or not. The documents may come from teaching and research institutions in France or abroad, or from public or private research centers.
L'archive ouverte pluridisciplinaire HAL, est destinée au dépôt et à la diffusion de documents scientifiques de niveau recherche, publiés ou non, émanant des établissements d'enseignement et de recherche français ou étrangers, des laboratoires publics ou privés. 


\title{
VAN HOVE SINGULARITIES AND CONTINUED FRACTION COEFFICIENTS
}

\author{
C. H. HODGES
}

C.N.R.S., Groupe des Transitions de Phases, B.P. 166, 38042 Grenoble Cedex, France

(Reçu le 9 février 1977, accepté le 28 mars 1977)

\begin{abstract}
Résumé. - On établit un rapport entre les singularités internes de Van Hove dans une bande et le comportement asymptotique des coefficients de la fraction continue qui décrit la densité d'états de cette bande. Ainsi, on peut démontrer que les coefficients oscillent autour de leurs bornes infinies avec une fréquence liée à la position de la singularité dans la bande, résultat déjà obtenu empiriquement par Gaspard et Cyrot-Lackmann. En outre, la méthode employée ici permet de déduire l'amplitude, la phase et la loi d'amortissement de ces oscillations.
\end{abstract}

\begin{abstract}
A relation is derived between the internal Van Hove singularities in a band and the asymptotic behaviour of the coefficients of the continued fraction which represents the density of states. Thus one may show that these coefficients oscillate about their infinite limit values with a frequency related to the position of the singularity within the band, a result first obtained empirically by Gaspard and Cyrot-Lackmann. The present method enables one to derive in addition the amplitude, phase and decay law of these oscillations.
\end{abstract}

The continued fraction [1] provides a powerful method of representing or approximating densities of states (e.g. for electrons or phonons) in periodic or aperiodic systems, without the necessity of calculating individual eigenvalues and eigenfunctions. It expresses the Green function of the system, whose imaginary part gives the density of states, in terms of real coefficients $a_{n}, b_{n}\left({ }^{1}\right)$, which may be calculated from a knowledge of the moments of the density of states [2] or by a process of tridiagonalising the Hamiltonian [3]. This method is applicable to a Hamiltonian (or dynamic matrix), expressed in a local, tightbinding representation, where the spectrum of eigenvalues is bounded and forms a band. It has recently been widely applied to the study of $\mathrm{d}$ bands in transition metals, both in the bulk and at the surface [e.g. 4, 5].

For an infinite (or semi-infinite) system, as $n \rightarrow \infty$ the coefficients $a_{n}$ and $b_{n}$ tend to limits $a_{\infty}, b_{\infty}$, which are related to the band extrema [3]. This permits the evaluation of the tail of the continued fraction which would give a smooth, continuous, semi-elliptical density of states. Structure is imposed on this density of states by the deviation for finite $n$ of the $a_{n}, b_{n}$ from

${ }^{1}$ ) In this article, the notation of reference [3] will be followed as far as possible. Note that the coefficient $b_{n}$ of reference [2] is equal to the square of $b_{n}$ in reference [3]. their asymptotic limits $a_{\infty}, b_{\infty}$. In particular, it is observed that internal Van Hove singularities in the band are related to the way in which $a_{n}, b_{n}$ approach their limits. Gaspard and Cyrot-Lackmann found empirically [2] by studying particular cases in three dimensions that the deviations

$$
a_{n}^{\prime}=a_{n}-a_{\infty}, \quad b_{n}^{\prime}=b_{n}-b_{\infty}
$$

oscillate with a frequency related to the position $E_{s}$ of the singularity and decay asymptotically at a rate governed by the sharpness of the singularity. Pottier has shown [6] how to derive this relation by treating the deviations $a_{n}^{\prime}, b_{n}^{\prime}$ as a perturbation on the semiinfinite chain $a_{n}=a_{\infty}, b_{n}=b_{\infty}$. Using a similar sort of idea, I shall show how to prove this result directly and very simply from the relations defining the continued fraction, and shall also derive quite generally the precise form of the asymptotic decay and its amplitude. I shall confine myself to a consideration of internal singularities in three dimensional structures.

As a function of complex energy $z$, the continued fraction is defined by :

$$
\begin{aligned}
R(z) & =b_{0}^{2} / x_{0} \\
x_{0} & =\left(z-a_{0}\right)-b_{1}^{2} / x_{1} \\
x_{1} & =\left(z-a_{1}\right)-b_{2}^{2} / x_{2}, \text { etc. } \\
x_{n} & =\left(z-a_{n}\right)-b_{n+1}^{2} / x_{n+1}, \text { etc. }
\end{aligned}
$$


The denominators $x_{n}(z)$ have the analytic properties :

$$
\begin{gathered}
\left|x_{n}(z)\right| \rightarrow \infty \text { as }|z| \rightarrow \infty \\
x_{n}\left(z^{*}\right)=x_{n}(z)^{*} \\
\operatorname{Im} x_{n}>0 \text { if } \operatorname{Im} z>0 .
\end{gathered}
$$

This gives $R(z)$, which is either the trace of, or a local diagonal matrix element of the Green function, the conventional analytic properties. In particular the density of states $N(E)$ which is positive is given by, [3],

$$
\begin{aligned}
N(E)=-\pi^{-1}[\operatorname{Im} R(z)]_{z} & =E+O i= \\
& =\pi^{-1}[\operatorname{Im} R(z)]_{z=E-O i} .
\end{aligned}
$$

The tail of the continued fraction may be summed by solving (1) in the asymptotic limit where $a_{n}=a_{\infty}$, $b_{n}=b_{\infty}$ and $x_{n}=x_{n+1}=x$ [3]. For energies lying within the band, $a_{\infty}-2 b_{\infty}<E<a_{\infty}+2 b_{\infty}, x$ is complex and given by

$$
\begin{aligned}
& x=b_{\infty} \mathrm{e}^{i \varphi} \\
& \varphi=\operatorname{arcos}\left(\frac{E-a_{\infty}}{2 b_{\infty}}\right),-\pi<\varphi<\pi .
\end{aligned}
$$

The two solutions of (4) are chosen to give the correct analytic properties (2). Thus

and

$$
\text { for } z=E+O i, \quad 0<\varphi<\pi
$$

$$
\text { for } z=E-O i, \quad-\pi<\varphi<0 .
$$

The relation between the Van Hove singularities and the continued fraction coefficients may be deduced by linearizing (1) in the asymptotic region, i.e. where the deviations $a_{n}^{\prime}, b_{n}^{\prime}$ and $x_{n}^{\prime}=x_{n}-x$ are small, but $n$ is finite. To first order in these deviations, one obtains :

$$
x_{n}^{\prime}=-a_{n}^{\prime}-2 \mathrm{e}^{-i \varphi} b_{n+1}^{\prime}+\mathrm{e}^{-2 i \varphi} x_{n+1}^{\prime},
$$

and its solution with the boundary condition $x_{\infty}^{\prime}=0$

$$
x_{n}^{\prime}=-\sum_{m=0}^{\infty} \mathrm{e}^{-2 m i \varphi}\left(a_{n+m}^{\prime}+2 \mathrm{e}^{-i \varphi} b_{n+m+1}^{\prime}\right) .
$$

Thus for $l>n$ and $n$ in the asymptotic region :

$$
\begin{aligned}
& a_{l}^{\prime}=-(2 \pi)^{-1} \int_{-\pi}^{+\pi} x_{n}^{\prime}(\varphi) \mathrm{e}^{(2 l-2 n) i \varphi} \mathrm{d} \varphi \\
& b_{l}^{\prime}=-(4 \pi)^{-1} \int_{-\pi}^{+\pi} x_{n}^{\prime}(\varphi) \mathrm{e}^{(2 l-2 n-1) i \varphi} \mathrm{d} \varphi .
\end{aligned}
$$

The asymptotic behaviour of $a_{l}^{\prime}, b_{l}^{\prime}(l \gg n)$ will be determined by the singularities in $x_{n}^{\prime}$, which, since $n$ is finite, must be at same energy as those of $R(z)$. Thus $x_{n}^{\prime}$ is singular at

$$
\varphi=\varphi_{\mathrm{s}}=\arccos \left(\frac{E_{\mathrm{s}}-a_{\infty}}{2 b_{\infty}}\right)
$$

and asymptotically $a_{l}^{\prime}, \quad b_{l}^{\prime}$ oscillate with a factor

$$
\cos \left(2 l \varphi_{\mathrm{s}}\right)=(-1)^{l} \cos w_{\mathrm{s}} l
$$

where

$$
w_{\mathrm{s}}=2 \arcsin \left(\frac{E_{\mathrm{s}}-a_{\infty}}{2 b_{\infty}}\right) .
$$

This is Gaspard's and Cyrot-Lackmann's result [2].

To determine the amplitude and rate of the asymptotic decay, it is necessary to relate the singularities in $x_{n}^{\prime}$ to those of $R(z)$. For a three dimensional Van Hove singularity $R(z)$ has a singular part

$$
\pm A \pi\left(E_{\mathrm{s}}-z\right)^{1 / 2} \text { or } \pm A \pi\left(z-E_{\mathrm{s}}\right)^{1 / 2}
$$

as may be verified from eq. (3), $A$ being the strength of the singularity. Using eq. (1), $R$ can be written as a function of $x_{n}=x+x_{n}^{\prime}$ and expanded thus :

$$
R=R_{n}\left(x+x_{n}^{\prime}\right) \simeq R_{n}(x)+\left(\frac{\mathrm{d} R}{\mathrm{~d} x_{n}}\right) x_{n}^{\prime}+\cdots
$$

where

$$
\frac{\mathrm{d} R}{\mathrm{~d} x_{n}}=-\prod_{m=0}^{n}\left[b_{m}^{2} / x_{m}^{2}\right]_{x_{n}=x} .
$$

For finite $n$, since $x$ is smooth, $R_{n}$ and $\mathrm{d} R / \mathrm{d} x_{n}$ are also smooth, and the singular part of $R$ is therefore contained in $x_{n}^{\prime}$. In the asymptotic limit $l \rightarrow \infty$, the Fourier transform eq. (7) is dominated by the square root singularity in $x_{n}^{\prime}$ which gives an $l^{-3 / 2}$ behaviour. One finds in this limit :

$$
\begin{aligned}
& a_{l}^{\prime} \rightarrow F l^{-3 / 2} \cos \left(2 l \varphi_{\mathrm{s}}+\eta\right) \\
& b_{l}^{\prime} \rightarrow \frac{1}{2} F l^{-3 / 2} \cos \left(2 l \varphi_{\mathrm{s}}-\varphi_{\mathrm{s}}+\eta\right)
\end{aligned}
$$

where

$$
F \mathrm{e}^{i \eta}=\mathrm{e}^{i \theta \frac{1}{2} A C}\left|\pi b_{\infty} \sin \varphi_{\mathrm{s}}\right|^{1 / 2}
$$

and :

$$
C=\frac{x_{0}^{2}}{b_{0}^{2}} L_{n \rightarrow \infty}^{t}\left[\prod_{m=1}^{n}\left(\frac{x_{m}}{x} \frac{b_{\infty}}{b_{m}}\right)^{2}\right]_{\varphi=\varphi_{\mathrm{s}}} .
$$

In eq. (12) $\theta$ takes the values $\pi / 4,-\pi / 4,3 \pi / 4$, $-3 \pi / 4$ corresponding to the four types of Van Hove singularity in three dimensions, $S_{2}$, minimum, $S_{1}$, maximum [7], respectively. Note the convergence of the constant $C$ in (13) with increasing $n$.

Relation (11) has been tested for the simple cubic s-band studied by Gaspard and Cyrot-Lackmann (the ferromagnetic case of ref. [2]). Extrapolating the coefficients $b_{l}$ given by these authors enables an estimate of $C$, the amplitude $F$ and phase $\eta$. The strength $A$ of the internal saddle point singularities has been given by Cyrot-Lackmann [8] for the simple cubic s-band. In figure 1 , the deviation

$$
\left[\left(b_{l} / b_{\infty}\right)^{2}-1\right] l^{3 / 2}
$$

obtained from the calculated values of $b_{l}^{2}$, [2], is compared to that given by eq. (11)-(13). This figure 


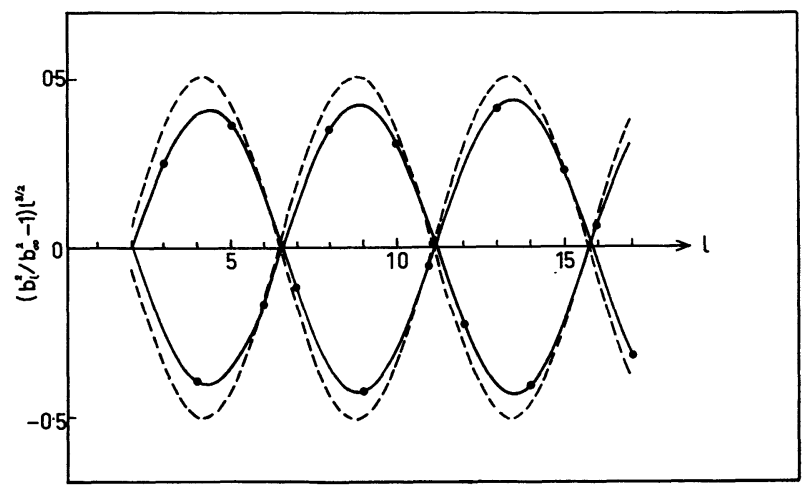

FIG. 1. - Shows the oscillatory behaviour of $\left(b_{l}^{2} / b_{\infty}^{2}-1\right) l^{3 / 2}$. The points (interpolated by the full line) are calculated using the values of $b_{l}$ given in ref. [2]. The dashed line represents the asymptotic behaviour given by eq. (11)-(13) of the text. The amplitude and phase of the oscillations are slowly approaching their asymptotic values with increasing $l$.

indicates that for $l<17$, the deviation has not yet reached its exact asymptotic amplitude and phase, but that the general $l^{-3 / 2}$ decay law is well obeyed.

In this work, the case of 2 and 1 dimensional internal singularities for which $R(z)$ has a divergent, logarithmic or inverse square root behaviour, has not been considered. Neither have singularities at the band edge $E_{0}$ when the density of states has the more general form $\left(E_{0}^{2}-E^{2}\right)^{v}$ with $v \neq 1 / 2$. For this latter case, the deviations $a_{l}^{\prime}, b_{l}^{\prime}$ do not oscillate since in eq. (11) $\varphi_{\mathrm{s}}=0$ or $\pi$. For such singularities Gaspard and Cyrot-Lackmann [2] showed $b_{l}^{2}$ to have the form $\left(0.25-v^{2}\right) b_{\infty}^{2} / l^{2}$ for large $l$. If $v$ is close to $1 / 2$, it is not difficult to show that the present method leads to the same result.

Some final remarks may be made concerning the use of eq. (11) to extrapolate the continued fraction coefficients. Since this equation applies to the linearized regime, the effect of different singularities will be a sum of terms of the type (11) with different values of $\varphi_{\mathrm{s}}, F$ and $\eta$. If the number of singularities is not too large, one might try to fit a series of coefficients $c_{n}$ by a sum over singularities $\alpha$

$$
c_{n}=\sum_{\infty} F_{\alpha} \cos \left(n \varphi_{\mathrm{s}, \alpha}+\eta_{\alpha}\right)
$$

where

$$
c_{n}=l^{3 / 2} a_{l}^{\prime} \quad \text { for } \quad n=2 l,
$$

and

$$
c_{n}=2 l^{3 / 2} b_{l}^{\prime} \text { for } n=2 l-1 .
$$

Whether such a scheme would be feasible for a case of physical interest remains to be seen.

Acknowledgements. - I am grateful to Roger Haydock who encouraged me to write up this work and to J. P. Gaspard and M. C. Desjonquères for useful discussions.

\section{References}

[1] WALL, H. S., Analytic Theory of continued fractions (Princeton, Van Nostrand) 1948.

[2] Gaspard, J. P. and Cyrot-Lackmann, F., J. Phys. C : Sol. St. Phys. 6 (1973) 3077.

[3] Haydock, R., Heine, V. and Kelly, M. J., J. Phys. C : Sol. St. Phys. 5 (1972) 2845 ; J. Phys. C : Sol. St. Phys. 8 (1975) 2591.
[4] Desjonquères, M. C. and Cyrot-Lackmann, F., J. Phys. F : Metal Phys. 5 (1975) 1368.

[5] Desjonquères, M. C. and Cyrot-Lackmann, F., J. Phys. $F$ : Metal Phys. 6 (1976) 567.

[6] Pottier, N., Doctoral thesis, Paris (1976) p. 31-4.

[7] Ziman, J. M., Principles of the theory of solids (Cambridge) 1964, p. 49.

[8] Cyrot-Lackmann, F., Doctoral thesis, Orsay (1968) p. 111. 